\title{
Fluid-structure interaction with a multiscale domain decomposition method
}

\author{
Etienne Vergnault*,** — Olivier Allix* — Serge Maison-le-Poëc** \\ * LMT Cachan \\ ENS Cachan/CNRS/Université Paris 6/PRES UniverSud Paris \\ 61 Avenue du Président Wilson, F-94230 Cachan \\ \{vergnault,allix\}@Imt.ens-cachan.fr \\ ** EADS Innovation Works \\ 12 rue Pasteur-BP 76, F-92152 Suresnes \\ serge.maison-le-poec@eads.net
}

\begin{abstract}
In order to solve structural problems submitted to a fluid-induced loading, we are interested in solving a fluid-structure interaction problem involving a deforming solid and a viscous fluid, under incompressible flow. We propose to use a partitioned but strongly coupled Gauss-Seidel scheme. We propose to solve the fluid part of the simulation by a finite element method with a scalable mixed domain decomposition strategy. A fictitious domain method enables taking into account the fluid-structure interface inside each fluid subdomain. Here, we describe the domain decomposition method and the coupling process on a stationary problem.

RÉSUMÉ. Afin de résoudre des problèmes de structures soumises à un chargement induit par un fluide, nous nous intéressons à la résolution d'un problème d'interaction fluide-structure impliquant un solide déformable et un fluide visqueux, en écoulement incompressible. Nous proposons d'utiliser un schéma partitionné mais fortement couplé de type Gauss-Seidel. Nous proposons de résoudre la partie fluide de la simulation par une méthode éléments finis avec une stratégie de décomposition de domaine mixte et extensible. Une méthode de domaine fictif permet de prendre en compte l'interface fluide-structure à l'intérieur de chaque sousdomaine fluide. Ici, nous décrivons les méthodes de décomposition de domaine et de couplage sur un problème stationnaire.

KEYWORDS: FSI, domain decomposition method, fictitious domain

MOTS-CLÉS : interaction fluide-structure, décomposition de domaine, méthode de domaine fictif.
\end{abstract}

DOI:10.3166/EJCM.19.267-280 (C) 2010 Lavoisier, Paris 


\section{Introduction}

The simulation of structures undergoing loading due to a fluid is one of the challenges in the aeronautical industry today. This paper reports the first steps toward the simulation of fluid-structure interaction (FSI) problems taking into account fine scale phenomena in the solid.

Composite materials are used by an always larger extent in structural parts and have degradation mechanisms at a very fine scale, compared to that of the structure. Multiscale domain decomposition methods (see, for eg. (Farhat et al., 1991; Ladevèze, 2004; Mandel, 1993)) are a powerful tool when facing problems with large number of degrees of freedom. The simulation of damage mechanisms in composite laminates using such methods is for example described in (Ladevèze, 2004). In order to take into account not only the prescribed external loads, but also those due to a transported fluid, we seek an extension of this method to fluid mechanics and FSI problems. Considering this particular context, and for the first developments, we restrict ourselves to fluid-structure interaction problems with the solid modeled as elastic in the finite deformation regime.

A lot of studies are devoted to coupled fluid-structure problems treated by partitioned methods. This choice is motivated by implementation facility, software reusability, and because it offers the possibility to adapt the numerical strategy to the problem at hand separately for the fluid and for the solid. We propose to use a classical partitioned but strongly coupled scheme, as proposed for example in (Wall et al., 1999). This Gauss-Seidel type of method solves two problems, one solid dynamics problem under prescribed fluid loading and one fluid dynamics problem with prescribed velocity at the interface.

The numerical resolution of fluid flow problems using finite element method has been extensively investigated (See reference books such as (Donea et al., 2003; Zienkiewicz et al., 2005) and references therein). We use a Eulerian description of the problem. Domain decomposition methods for fluid flow have been developed by many authors. To cite some, reference (Farhat et al., 2000) deals with aeroacoustic problems while (Toselli, 2001) describe a FETI method for scalar advection-diffusion equations. Here, like what is done in domain decomposition for incompressible solid mechanics (Gosselet et al., 2002; Vereecke et al., 2003), the incompressibility is taken into account with a mixed formulation. This is classical for incompressible fluid flows: in (Li, 2002), FETI algorithms are adapted to incompressible Stokes and linearized Navier-Stokes equations. In this study, we focus on stationary viscous incompressible flow. The non-linearity arising from the convective term of the Navier-Stokes equations is treated at the subdomain level, instead of being linearized at the global level. The sub-domain problems are not condensed on the interfaces, nor preconditioned, but a macroproblem can be added in the strategy to improve its convergence rate.

We first expose the general fluid-structure interaction problem. As we propose to use a partitioned strategy, we first focus rapidly on the resolution of the fluid problem alone, by a mixed domain decomposition method. Then, taking into account the pres- 
ence of a solid inside the flow is equivalent to adding a new interface, similar to the ones in the fluid domain decomposition. We farther discuss the appropriateness of the different choices we made with some examples.

\section{Fluid-structure interaction problem statement}

In this paper we consider the interaction of a fluid and a solid, along the interface $\Gamma_{c}$. The fluid occupies the domain $\Omega$, and the solid domain is called $\omega$ with boundary $\partial \omega$. We assume that there is only one solid body, and only one fluid in $\Omega$. The boundary of the solid is either submitted to prescribed displacement or wet.

The Gauss-Seidel scheme is equivalent to a strong coupling scheme but partitioned (Matthies et al., 2002). The solution is sought iteratively, by solving the fluid problem with prescribed interface position and velocity for forces, and by solving the solid problem with prescribed forces for interface velocity and position.

\section{Resolution of the fluid problem by a domain decomposition method}

In the cases we have in mind, the fluid domain may extend to a much larger domain than the solid one. However, the descriptions near the interface must be of comparable characteristic size to ensure good quality results. Due to the fact that we aim at computing quite precisely the effect of the fluid on the solid, the fluid problem itself must be finely discretized, that is why we have sought a scalable domain decomposition method for the fluid. Here, we only state the decomposition of the domain and the resolution of the decomposed fluid problem. Convergence and scalability are discussed in more details in (Vergnault, 2009).

\subsection{Fluid problem statement}

The fluid is assumed Newtonian, with kinematic viscosity $\nu$ and density $\rho$. The flow in $\Omega$ is considered as incompressible, modeled by the Navier-Stokes equations, formulated with velocity $\underline{v}$ and pressure $p$ unknowns. The flow is submitted to prescribed velocity $\underline{v}_{d}$ on $\partial_{\underline{v}} \Omega$ and prescribed traction $\underline{f}$ on the complementary part $\partial_{f} \Omega$ of $\partial \Omega$. We focus in this paragraph on the treatment of the fluid problem alone.

After integration by parts, the weak form of the Navier-Stokes problem becomes: Find $(\underline{v}, p)$ in suitable spaces such that $\forall(\underline{w}, q)$ :

$$
(\underline{w}, \underline{v \nabla v})_{\Omega}+\left(\underline{\underline{\nabla}}^{S} \underline{w}, 2 \nu \underline{\underline{\nabla}}^{S} \underline{v}\right)_{\Omega}-(p, \underline{\nabla} \cdot \underline{w})_{\Omega}-(q, \underline{\nabla} \cdot \underline{v})_{\Omega}=(\underline{f}, \underline{w})_{\partial_{\underline{f}} \Omega}
$$

where $(,)_{D}$ is the scalar product over domain $D$.

Note that Equation [1] has been obtained through what is called the velocity-stress divergence form of the momentum equation, and $f$ are the prescribed boundary tractions. 


\subsection{Reformulation through domain decomposition}

Domain $\Omega$ is split into sub-domains $\Omega_{E}$. These subdomains are disjoint and connected by interfaces. Interface $\Gamma_{i j}$ connects Sub-domains $\Omega_{i}$ and $\Omega_{j}$. These interfaces can also connect the subdomains to the boundary conditions. We choose as variables the velocity and pressure fields $\underline{v}_{E}$ and $p_{E}$ on the sub-domains and distributions of velocity and effort $\underline{W}_{E}$ and $\underline{F}_{E}$ on the edges of the subdomains. $\underline{\underline{\sigma}}_{E}=-p_{E} \underline{n}+\nu \underline{n} \cdot \underline{\underline{\nabla}}^{S} \underline{v}_{E}$ is the Cauchy stress tensor on $\Omega_{E}$. The problem can then be reformulated as follows:

Find for each sub-domain the velocity and pressure fields $\underline{v}_{E}$ and $p_{E}$, and the edge fields $\underline{W}_{E}$ and $\underline{F}_{E}$ such that:

- Point 1: $\underline{v}_{E}$ and $p_{E}$ are solution of the Navier-Stokes equations, with mixed boundary conditions and $\underline{W}_{E}=\left.\underline{v}_{E}\right|_{\partial \Omega_{E}}$ and $\underline{F}_{E}=\left.\underline{\sigma}_{E} \underline{n}\right|_{\partial \Omega_{E}}$ rium

- Point 2: fields $\underline{W}_{E}$ are continuous between subdomains and $\underline{F}_{E}$ are in equilib-

\subsection{Resolution by an iterative strategy}

The two points outlined above are the basis of the iterative strategy used for solving the problem. The iterative process is a procedure which constructs approximations verifying alternatively each set of equations. Those sets are respectively represented by a curve and a straight line on Figure 1. Starting from data on the interfaces, we seek a subsequent approximation in the subdomains by following Search direction $Z-$ and get back to a an approximation on the interfaces with Search direction $Z+$. The solution of the problem is at the intersection of the curve and the line, where interface fields are balanced and interior fields verify the Navier-Stokes equations.

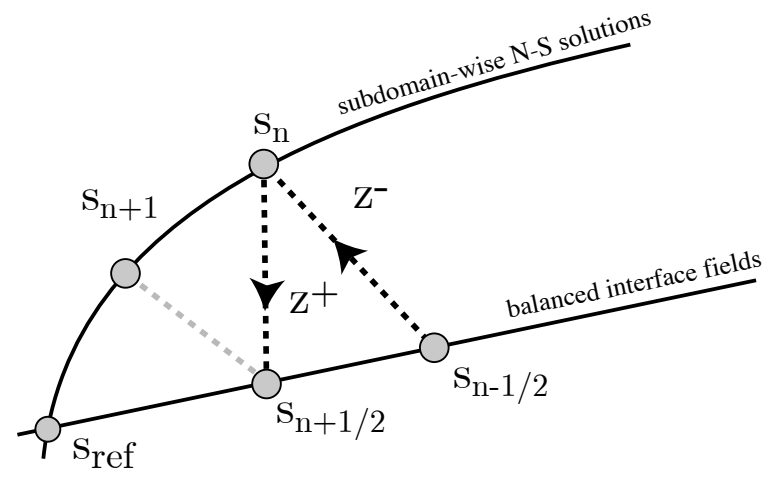

Figure 1. Schematic representation of the strategy 


\subsubsection{The interface stage}

The interfaces connect subdomains edges to one another. Each interface has two sides corresponding to the neighboring subdomain's edges, whose quantities are denoted $\underline{W}, \underline{F}$ and $\underline{W}^{\prime}, \underline{F}^{\prime}$. At the interface stage, we designate the unknown velocity and forces with a hat: $\underline{\widehat{W}}, \underline{\underline{F}}$. They have to verify continuity and equilibrium and are related by a search direction equations on each side: $\underline{\widehat{F}}-\underline{F}-\underline{z}(\underline{\widehat{W}}-\underline{W})=\underline{0}$ and $-\underline{\widehat{F}}-\underline{F^{\prime}}-\underline{z}\left(\widehat{W}-\underline{W^{\prime}}\right)=\underline{0}$. The solution of this interface stage is thus readily accessible: $\widehat{\widehat{W}}=\frac{1}{2}\left(\underline{W}+\underline{W^{\prime}}\right)-\frac{1}{2} \underline{z}^{-1}\left(\underline{F}+\underline{F^{\prime}}\right)$ and $\underline{\widehat{F}}=\frac{1}{2}\left(\underline{F}-\underline{F^{\prime}}\right)-\frac{1}{2} \underline{z}\left(\underline{W}-\underline{W^{\prime}}\right)$. The boundary conditions are treated as a special type of interface, since the equation of search direction is replaced by the given effort or velocity on the corresponding side of the interface. $\underline{z}$ is the search direction parameter. It can be determined from fluid viscosity and interface characteristic length. Numerical experiments showed that the best convergence rate was for $\underline{\underline{z}}=10 \frac{\nu \rho}{L} \underline{\underline{I}}$ with $L$ the length of the interface.

\subsubsection{The subdomain stage}

Starting from a solution $\left(\widehat{W}_{E}, \widehat{F}_{E}\right)$ on the interfaces connected to each subdomain, we seek a solution $\left(\underline{v}_{E}, p_{E}, \underline{W}_{E}=\left.\underline{v}_{E}\right|_{\partial \Omega_{E}}, \underline{F}_{E}\right)$ of the Navier-Stokes equation on each subdomain. With the search direction $\left.\left(\underline{F}_{E}-\underline{\widehat{F}}_{E}+\underline{\underline{z}}_{\underline{W}}-\underline{W}_{E}\right)=0\right)$, the problem writes:

$$
\begin{aligned}
& \int_{\Omega_{E}} \underline{w}_{E} \cdot\left(\underline{v}_{E} \underline{\underline{\nabla v}} E\right)+2 \nu \underline{\underline{\nabla}}^{S} \underline{v}_{E}: \underline{\underline{\nabla}}^{S} \underline{w}_{E}-p_{E} \underline{\nabla} \cdot \underline{w}_{E}-q_{E} \underline{\nabla} \cdot \underline{v}_{E} \mathrm{~d} \Omega \\
& +\int_{\partial \Omega_{E}} \underline{z W}=\underline{W}_{E}^{*} \mathrm{~d} S=\int_{\partial \Omega_{E}}\left(\widehat{\widehat{F}}_{E}+\underline{\underline{z W}}_{E}\right) \cdot \underline{W}_{E}^{*} \mathrm{~d} S
\end{aligned}
$$

Equation [2] is then stabilized by standards SUPG/PSPG methods (Brooks et al., 1982; Tezduyar et al., 1992) and linearized by a Newton-Raphson scheme.

REMARK. - Scalability

Exposed as is, the convergence of the iterative strategy depends strongly on the number of subdomains used in the partition of $\Omega$. This severe drawback can be alleviated by prescribing volume conservation on each subdomain in terms of $\widehat{W}$. The interface stage should be consequently modified, in order to incorporate an additional problem, called macroproblem. As scalability is not the aim of this contribution, this subject will not be discussed any further here. In the following, this domain decomposition method with the macroproblem is named $M u S$.

\section{Taking into account the fluid-structure interface}

This section is dedicated to the coupling algorithm of the fluid flow with the solid $\omega$, along the boundary $\Gamma_{c} . \Omega$ is still the computational fluid domain, but the real flow takes place in $\Omega^{+}$, the part of $\Omega$ that is not overlaid by $\omega$, while $\Omega^{-}$is the overlaid 
part. Figure 2 clarifies the notations. $\Omega^{+}$is called the real part of the flow, while $\Omega^{-}$ is the fictitious domain. The domain decomposition is applied to $\Omega$, so there can be several fluid subdomains involved in the interaction with the structure.

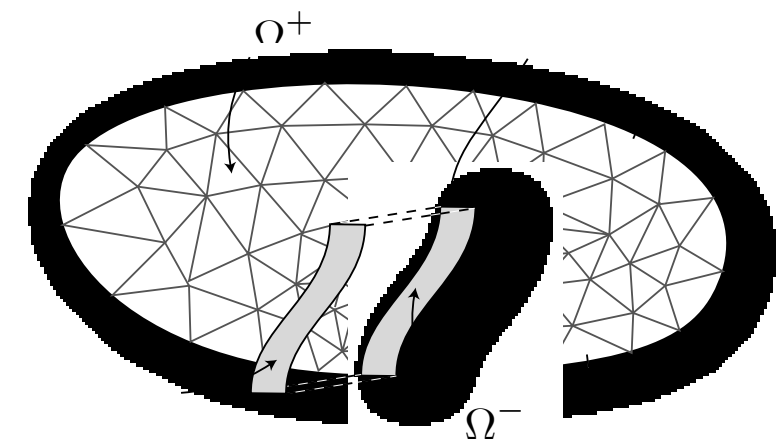

Figure 2. Fluid-structure interaction notations

\subsection{Mixed coupling interfaces}

The mixed interfaces defined in the previous section can be used as fluid-solid interfaces. They hold the same velocity and force distributions $\underline{W}$ and $\underline{F}$ that the perfect interfaces between two fluid sub-domains. The coupling is done similarly to what is done in the Fictitious Domain Method (Glowinski et al., 1997; Yu, 2005), but with mixed interfaces. These mixed interfaces are used to enforce boundary conditions on the boundary of the fluid domain in the previous section. We propose here to extend their capabilities to enforce boundary conditions, inside the fluid domain, along the fluid-solid interface. They hold boundary conditions for the real part of the flow, along a surface that is not explicitly described by the fluid mesh. In this, the method used here is similar to the fictitious domain method, except that no Lagrange multipliers are used. The same iterative strategy than the one previously used can then be adapted to this novel situation with a prescribed velocity on the interface for the fluid: $\widehat{W}_{\Gamma_{c}}=\underline{W}_{s}$. Thus the search direction equation will be the same as for BC interfaces in the previous section and the interface stage is not modified. The balance of forces is achieved iteratively through the Gauss-Seidel coupling scheme. After the fluid resolution, the solid is submitted to fluid forces $\widehat{F}_{\Gamma_{c}}=\underline{F}_{s}$ on the interface. The differences between the $M u S$ method and that one $(M u S+F S I)$ are in the subdomain stage.

\subsection{Modification of the sub-domain stage}

The sub-domain stage has to be modified only for the fluid sub-domains involved in the coupling, in order to take into account the new coupling interface. Let $\Omega_{E}$ be a 
subdomain and drop ${ }_{E}$ subscripts, so $\underline{v}$ and $p$ are the velocity and pressure fields inside it.

Starting from $\left(\widehat{\underline{W}}_{E}, \widehat{\underline{F}}_{E}\right)$ on the interfaces and from $\left(\widehat{\underline{W}}_{\Gamma_{c}}, \widehat{\underline{F}}_{\Gamma_{c}}\right)$ on the part of $\Gamma_{c}$ intersecting the subdomain at hand we want to compute $\left(\underline{v}, p, \underline{W}_{E}=\left.\underline{v}\right|_{\partial \Omega_{E}}, \underline{F}_{E}=\right.$ $\left.\left.\underline{\sigma} \underline{\underline{n}}\right|_{\partial \Omega_{E}}, \underline{W_{\Gamma_{c}}}=\left.\underline{v}\right|_{\Gamma_{c}}, \underline{F}_{\Gamma_{c}}=\left.\underline{\sigma}_{E} \underline{n}\right|_{\Gamma_{c}}\right)$, solution of the Navier-Stokes equations under mixed boundary conditions. The problem writes the same as in Equation [2] except that there are two extra integrals to compute. The left-hand term should be added $\int_{\Gamma_{c}} \underline{z} W_{\Gamma_{c}} \cdot \underline{W}_{\Gamma_{c}}^{*} \mathrm{~d} S$ while the right-hand term should be $\int_{\partial \Omega_{E}}\left(\underline{\widehat{F}}_{E}+\underline{\underline{z}}_{E}\right)$. $\underline{W}_{E}^{*} \mathrm{~d} S+\int_{\Gamma_{c}}\left(\widehat{\underline{F}}_{\Gamma_{c}}+\underline{z} \widehat{W}_{\Gamma_{c}}\right) \cdot \underline{W}_{\Gamma_{c}}^{*} \mathrm{~d} S$. We use a mortar-like projection method to compute these interface integrals. The projections links $\underline{v}_{E}$ to $\underline{W}_{\Gamma_{c}}$ and $\underline{W}_{E}$.

The coupling interface mesh is not included in the mesh of the neighboring fluid subdomain. Hence, field extraction from the sub-domain to the interface is more complicated than for perfect fluid-fluid interfaces. Figure 3 shows the difference between the two types of interfaces. In the subdomain problem, the integrals along $\Gamma_{c}$ are computed on the skin mesh of the solid, after projecting the fluid fields on it. This projection is achieved by matrices built to project the fluid nodes position onto the interface nodes ones.

\section{Subdomain stiffness matrix}

The finite element equivalent of Equation [2] is to find $U_{E}$, a vector containing the fluid degrees of freedom, solution of the matrix problem: $U_{E}^{* T} K U_{E}=U_{E}^{* T} F_{g}$ where $F_{g}$ is the generalized force vector. The assembly of the Navier-Stokes part of Matrix $K$ is a standard in FE methods, we report here the assembly of the boundary terms, involving interface fields. For each interface $\Gamma_{i}$ of $\partial \Omega_{E}$ (There is one for each neighbor of $\Omega_{E}$ plus one for each boundary condition), we define a stiffness matrix such that $\int_{\Gamma_{i} \in \partial \Omega_{E}} \underline{z} W_{E, i} \cdot \underline{W}_{E, i}^{*} \mathrm{~d} S=U_{E}^{* T} K_{\Gamma_{i}} U_{E}$. Similarly, the fluidsolid interface is attributed a stiffness matrix such that: $\int_{\Gamma_{c}} \underline{z} W_{\Gamma_{c}} \cdot \underline{W}^{*} \Gamma_{c} \mathrm{~d} S=$ $U_{E}^{* T} K_{\Gamma_{c}} U_{E}$.

For the perfect interfaces, the stiffness matrix is computed from the projection and integration matrices $N_{i}$ and $M_{i}: W_{E, i}=N_{E, i} U_{E}$ and $\int_{\Gamma_{i}} \underline{z} W_{E, i} \cdot \underline{W}_{E, i}^{*} \mathrm{~d} S=$ $U_{E}^{* T} \underline{\underline{z}} N_{E, i}^{T} M_{E, i} N_{E, i} U_{E}$. The matrices $M_{E, i}$ and $N_{E, i}$ are computed while initializing the domain decomposition and stored. As the interface fields are constant on each element, these matrices are rather straightforward to compute, since $M_{E, i}$ is a diagonal matrix containing the interface elements lengths and $N_{E, i}$ projects node values on elements-centered points. Matrix $N_{E, i}$ size is kept small, of the order of the number of nodes in the interface to the power two, by using an index for interface nodes: $W_{E, i}=N_{E, i} U_{E}\left[\right.$ index $\left._{i}\right]$.

For the fluid-solid interfaces, we do exactly the same, using the solid skin mesh as interface mesh. Matrix $N_{\Gamma_{c}}$ thus links $U_{E}$ to $W_{\Gamma_{c}} . M_{\Gamma_{c}}$ is computed from the interface elements lengths, and $N_{\Gamma_{c}}$ is a submatrix of the minimization problem: Given $\underline{u}$, find $\underline{U}$ on $\Gamma_{c}$ minimizing $E_{p}(\underline{U}, \underline{u})=\int_{\Gamma_{c}}\|\underline{U}-\underline{u}\|^{2} \mathrm{~d} S$. The associated matrix 
problem writes: $\left[\begin{array}{ll}-B^{T} & M_{\Gamma_{c}}\end{array}\right]\left[\begin{array}{ll}u & U\end{array}\right]^{T}=0$ and thus: $U=M_{\Gamma_{c}}^{-1} B^{T} u=N_{\Gamma_{c}} u$. Matrix $B$ is assembled from element contributions. We use symbolic derivation on the minimization problem to get an expression of element-wise $B$ that depends only on the position of interface nodes. Eventually, we obtain a generic builder for Matrix $N_{\Gamma_{c}}$ that depends only on the nodes position. Obviously, the same indexing as for perfect interfaces occurs, and the size of $N_{\Gamma_{c}}$ is lowered: $W_{\Gamma_{c}}=N_{\Gamma_{c}} U_{E}\left[\operatorname{index}_{\Gamma_{c}}\right]$

The right hand terms in the subdomain problem are assembled in the same fashion:

$$
U_{f}^{* T} F_{g}=U_{f}^{* T}\left(\tilde{N}_{i} N_{E, i} M_{E, i}\left(\underline{\widehat{F}}_{E, i}+\underline{z} \widehat{\widehat{W}}_{E, i}\right) \tilde{N}_{\Gamma_{c}} N_{\Gamma_{c}} M_{\Gamma_{c}}\left(\underline{\widehat{F}}_{\Gamma_{c}}+\underline{z}^{\underline{W_{W}}} \Gamma_{c}\right)\right)
$$

$\tilde{N}$ denotes boolean matrices for the assembly of contributions. They are never constructed nor stored, but are used to write formally the assembly process.

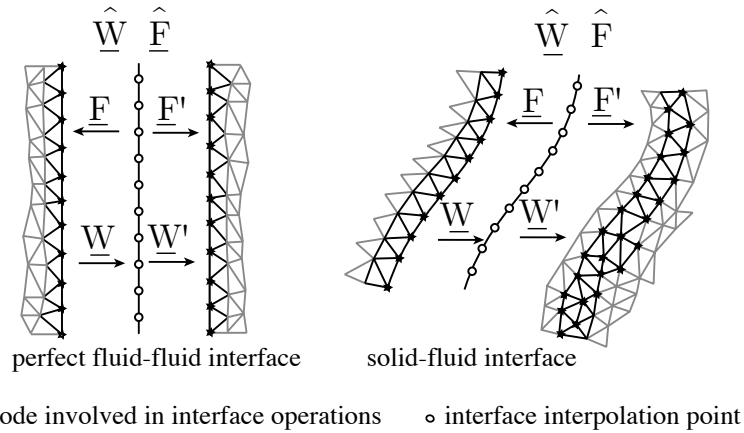

Figure 3. Mixed interfaces: perfect fluid-fluid and fluid-solid interfaces

\section{Examples}

\subsection{Fixed interface over a fixed mesh: flow around a cylinder}

The flow around a cylinder benchmark is used to assess the capabilities of the fluidsolid interface. We take as a reference the solution computed with the $M u S$ method, on a mesh compatible with the solid boundary.

A cylinder of diameter 0.1 is set at position $(0.2,0.2)$ between two plates $(0 \leq x \leq$ $2.2, y=0$ and $y=0.41)$. The velocity on the inflow $(x=0)$ is prescribed parabolic and the outflow is free. Based on the cylinder diameter and mean inflow velocity, the Reynolds number is 20 . The fluid domain is decomposed in 88 sub-domains. In order to reduce the differences between the $M u S$ results and the $M u S+F S I$ results, The fluid domain decomposition used is the same, and the meshes for the fluid sub-domains that are not involved in the coupling are the same. The local mesh size around the interface is twice smaller in the $M u S+F S I$ case. Using continuous interpolation for 
fluid fields through the interface perverts the solution in the MuS+FSI method. To obtain a similar precision in the aerodynamical coefficients, we used a locally refined mesh. Fluid subdomains are not interconnected in the fictitious part of the flow. As there is no interest in that part of the flow, there is no need to converge the domain decomposition solution inside it.

In this particular case, coupling the fluid flow and the solid reduces to impose zerovelocity boundary condition on a line that is not included in the fluid mesh. In order to quantify the error introduced by the coupling interface, some coefficients are extracted from the results and compared to those obtained with the $M u S$ method and by other authors.
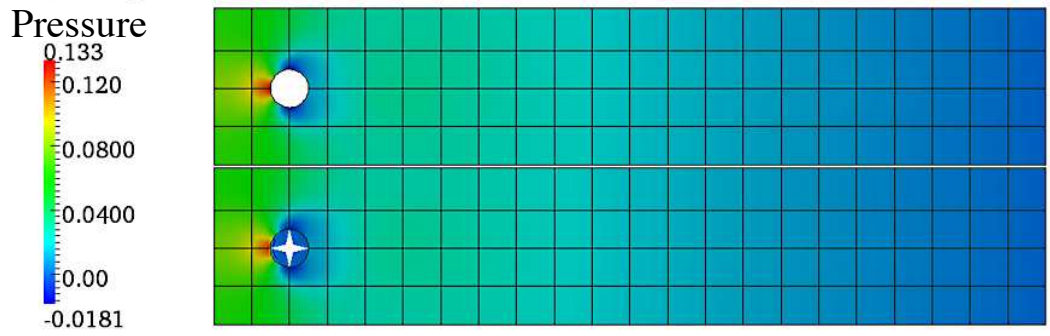

Figure 4. Pressure field. MuS method (top), MuS+FSI method (bottom)

$$
\begin{gathered}
\text { velocity } \\
0.404 \\
0.400 \\
0.300 \\
0.200 \\
0.100 \\
6.92 \mathrm{e}-05
\end{gathered}
$$

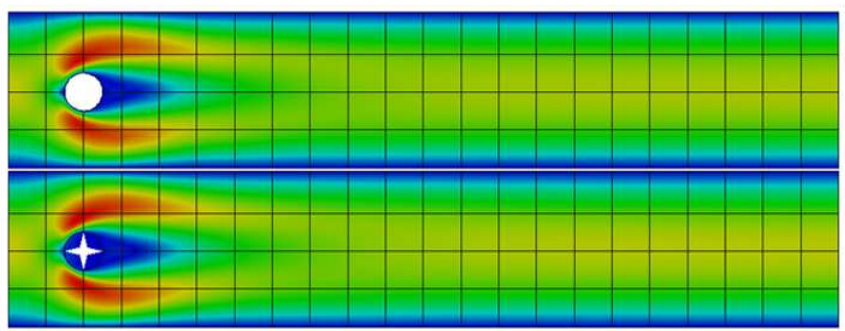

Figure 5. Velocity field. MuS method (top), MuS+FSI method (bottom)

\section{Quality of the results}

Figures 4 and 5 draw the computed velocity and pressure fields, after 50 iterations of the fluid domain decomposition method, with fluid-structure interaction. They are compared to the fields computed using an explicit description of the interface. The two methods achieve similar results, the only major differences being in the fluid elements intersected by the fluid-solid interface $\Gamma_{c}$. As only continuous pressure and velocity interpolations are used, the $M u S+F S I$ method cannot capture the physical discontinuities in the problem. This is a classical drawback in the fictitious domain 
Table 1. Comparison of computed aerodynamic coefficients and reference ones

\begin{tabular}{|l|l|c|c|c|c|}
\hline method & & $c_{D}$ & $c_{L}$ & $\Delta p$ & $l_{r}$ \\
\hline$M u S$ & $88 \mathrm{sd}$ & 5.65 & 0.013 & 0.1183 & 0.85 \\
$M u S+F S I$ & $88 \mathrm{sd}$ & 5.70 & 0.018 & 0.1186 & 0.80 \\
\hline & lower bound & 5.57 & 0.011 & 0.12 & 0.85 \\
reference & upper bound & 5.59 & 0.011 & 0.12 & 0.85 \\
\hline
\end{tabular}

method, and in embedded methods in general. Results can be improved by using a local enrichment, for example by X-FEM (Gerstenberger et al., 2008). As this is not done yet the continuous interpolations through the fluid-solid interface introduces an additional error.

Table 1 exposes the computed results and the reference values. With both methods, the drag $\left(c_{D}\right)$ and lift $\left(c_{L}\right)$ coefficients, the pressure drop $(\Delta p)$ and the length of the recirculation zone $\left(l_{r}\right)$ are in good agreement with the results collected by (Schäfer $e t$ al., 1996).

\subsection{Moving interface over a fixed mesh: channel flow with a flap}

Consider now a plane Couette flow problem, with a flexible wall between the two plates. This wall is assumed to have an elastic behavior, and to undergo finite strain. The prescribed velocity on the left of the channel is kept small, so that the Reynolds number is low (about 10) and the flow stays stationary. This example is designed to test the Gauss-Seidel strong coupling scheme with mixed interfaces and large structural displacement, over one coupling step.

Figure 6 shows the wall tip displacement along coupling iterations. Rapidly, the structure gets to a deformation close to the equilibrium one. Figure 7 represents the deformed configuration after the 13 iterations to achieve the convergence criterion. Color fields are the velocity magnitude on the fluid domain and Von Mises stress on the solid mesh.

Due to the large displacement of the structure, the underlying fluid mesh has to be fine enough in an extended region. Indeed, the former example showed that the accuracy of the results would not be satisfying with a large fluid mesh size in the coupling zone. This is a known drawback of fixed mesh methods for fluid-structure interaction. Remedy to that include adaptive meshes (van Loon et al., 2004), or local enrichment strategies.

When used with a moving structure, the mixed interfaces need a supplementary step. Field exchange on the coupling interface is achieved through matrices, linking the solid quantities, on the solid interface nodes to the fluid ones, on a cloud of nodes. As the interface moves, the fluid nodes in the cloud involved in the data transfer change, and the matrices need to be actualized. This implies a call to the matrix builder 
at each Gauss-Seidel step, hence a loop on all fluid nodes in the sub-domain. As in the coupling zone, the fluid mesh has to be rather fine, the matrix builder is potentially slow. But this interface capturing step is mandatory in fixed fluid mesh strategies, and there is no way to avoid it, except using a moving mesh, what we excluded from the beginning.

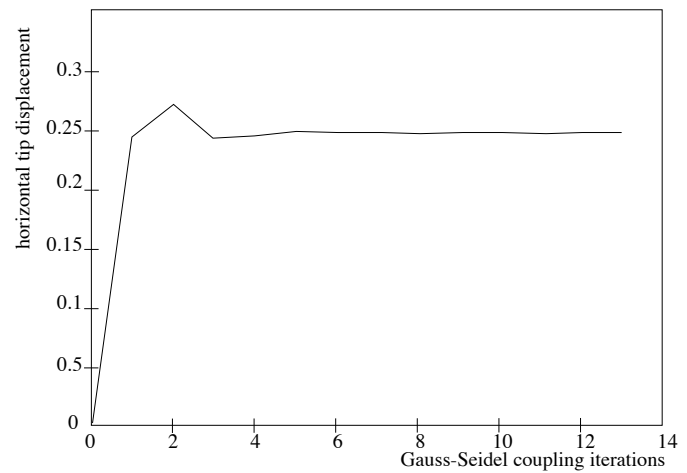

Figure 6. Tip displacement versus Gauss-Seidel coupling iterations

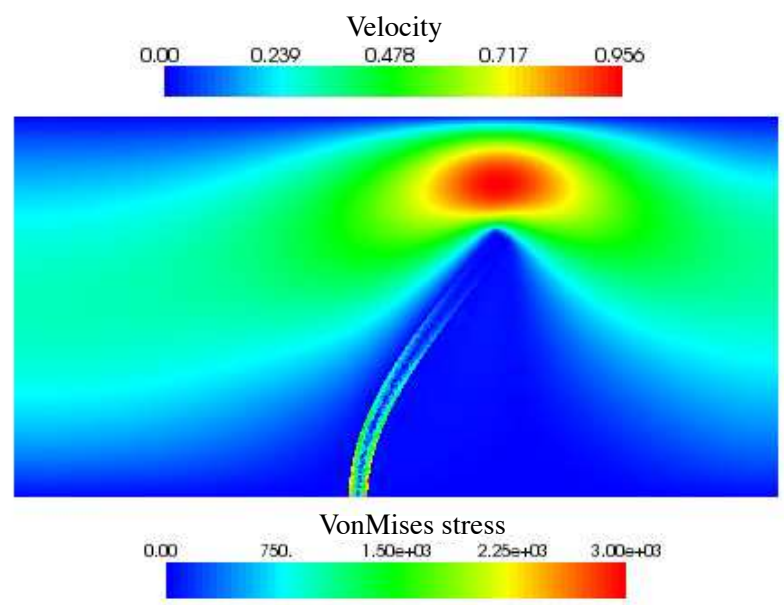

Figure 7. Convergence criterion is reached at Iteration 13

Then, when the coupling interface comes to crossing a fluid-fluid interface, all the coupling interface objects should be actualized. As some solid nodes on the interface 
come over a fluid sub-domain which is different from the one they were over in the last step, the interface meshes themselves have to be rebuilt. Failure to do so leads to no coupling along some parts of the solid boundary, as shown on Figure 8. The coupling interfaces are created from the initial configuration. Due to the large displacements of the solid, some nodes on its boundary are overlying a fluid sub-domain they are not coupled to, and leaks through the solid appear. This potential shift of a solid node between two fluid sub-domains implies a deeper actualization than in the previous paragraph:

- detect the fluid sub-domain corresponding to each node on the solid boundary (from node position and fluid sub-domain box)

- create as many coupling interfaces as fluid sub-domains involved in the coupling

- build a mesh for each coupling interface (from the solid nodes)

- compute the coupling matrix for each interface

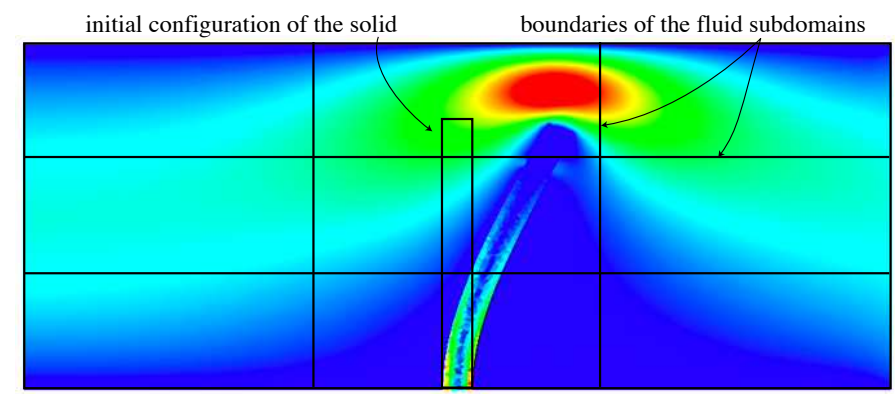

Figure 8. Solid boundary nodes shifting between fluid sub-domains results in leaks through the interface

\section{Conclusion}

The combination of an iterative strategy to solve the fluid problem with prescribed boundary conditions and a Gauss-Seidel fluid-structure coupling scheme makes each FSI step potentially expensive (hence each time-step in a transient simulation), and the strategy proposed here should be compared to its counterpart, using Block-Newton coupling strategy and a moving fluid mesh, tied to the solid at the interface. The last should be cheaper in terms of iterations, but each iteration much more expansive than in the former. We have nevertheless demonstrated the feasibility of the method, and stressed its weaknesses. 
FSI with DDM 279

\section{Acknowledgements}

This research was supported by EADS Innovation Works.

\section{References}

Brooks A. N., Hughes T. J. R., “ Streamline upwind/Petrov-Galerkin formulations for convection dominated flows with particular emphasis on the incompressible Navier-Stokes equations", Computer Methods in Applied Mechanics and Engineering, vol. 32, n 1-3, p. 199259, 1982.

Donea J., Huerta A., Finite Elements Methods for Flow Problems, Wiley, 2003.

Farhat C., Lesoinne M., " Two efficient staggered algorithms for the serial and parallel solution of three-dimensional nonlinear transient aeroelastic problems", Computer Methods in Applied Mechanics and Engineering, vol. 182, n 3-4, p. 499-515, 2000.

Farhat C., Roux F., " A method of Finite Element Tearing and Interconnecting and its parallel solution algorithm", International Journal for Numerical Methods in Engineering, vol. 32, p. 1205-1227, 1991.

Gerstenberger A., Wall W., “ An extended finite element / mortar method based approach for fluid-structure interaction", Computer Methods in Applied Mechanics and Engineering, vol. 197, p. 1699-1714, 2008

Glowinski R., Pan T. W., Periaux J., “ A Lagrange multiplier/fictitious domain method for the numerical simulation of incompressible viscous flow around moving rigid bodies: (I) case where the rigid body motions are known a priori", Comptes Rendus de l'Académie des Sciences, vol. 324, p. 361-369, 1997.

Gosselet P., Rey C., Léné F., Dasset P., “ A domain decomposition method for quasiincompressible formulations with discontinuous pressure field - Application to the mechanical study of a flexible bearing", Revue Européenne des Eléments Finis, vol. 11, p. 363-378, 2002.

Ladevèze P., " Multiscale modelling and computational strategies for composites", International Journal for Numerical Methods in Engineering, vol. 60, n 1, p. 233-253, 2004.

Li J., Dual primal FETI methods for stationary Stokes and Navier-Stokes equations, $\mathrm{PhD}$ thesis, Courant Institute of Mathematical Sciences, september, 2002.

Mandel J., "Balancing domain decomposition", Communications in Numerical Methods in Engineering, vol. 9, p. 233-241, 1993.

Matthies H., Steindorf J., " Partitioned but strongly coupled iteration schemes for nonlinear fluid-structure interaction”, Computer and Structures, vol. 80, p. 1991-1999, 2002.

Schäfer M., Turek S., “ Benchmark computations of laminar flow around a cylinder", Flow Simulation with High-Performance Computation II, vol. 52, p. 547-566, 1996.

Tezduyar T. E., Mittal S., Ray S. E., Shih R., “Incompressible flow computations with stabilized bilinear and linear equal-order-interpolation velocity-pressure elements", Computer Methods in Applied Mechanics and Engineering, vol. 95, n² 2, p. 221-242, 1992.

Toselli A., " FETI domain decomposition methods for scalar advection-diffusion problems", Computer Methods in Applied Mechanics and Engineering, vol. 190, $\mathrm{n}^{\circ} 43-44$, p. 57595776, 2001. 
van Loon R., Anderson P., De Hart J., Baaijens F. P. T., “ A combined fictitious domain/adaptive meshing method for fluid-structure interaction in heart valves", International Journal for Numerical Methods in Fluids, vol. 46, n 5, p. 533-544, 2004.

Vereecke B., Bavestrello H., Dureisseix D., " An extension of the FETI domain decomposition method for incompressible and nearly incompressible problems", Computer Methods in Applied Mechanics and Engineering, vol. 192, n 31-32, p. 3409-3429, 8, 2003.

Vergnault E., Vers une approche multi-échelle pour l'interaction Vers une approche multiéchelle pour l'interaction fluide-structure, $\mathrm{PhD}$ thesis, ENS Cachan, 2009.

Wall W., Mok D., Ramm E., “ Partitioned Analysis Approach for the Transient, Coupled Response of Viscous Fluids and Flexible Structures", ECCM'99-Proc. European conference on computational mechanics, Munich, Germany, August 31-September 3, 1999.

Yu Z., " A DLM/FD method for fluid/flexible-body interactions", Journal of Computational Physics, vol. 207, n 1, p. 1-27, 2005.

Zienkiewicz O., Taylor R., Nithiarasu P., The Finite Element Method for Fluid Dynamics, Butterworth-Heinemann, 2005. 